\title{
Grape Quality Mapping for Vineyard Differential Harvesting
}

\author{
Antonio Odair Santos ${ }^{*}$, Robert L. Wample ${ }^{2}$, Sivakumar Sachidhanantham ${ }^{2}$ and Oren \\ Kaye $^{3}$ \\ ${ }^{1}$ Instituto Agronômico; Rodovia Dom Gabriel Paulino Bueno Couto, Km 65, CEP 13212-240; Jundiaí - SP - Brasil. \\ ${ }^{2}$ Viticulture and Enology Research Center; California State University; 2360 East Barstow Avenue, Fresno - CA - \\ USA. ${ }^{3}$ Constellation Wines, 12667 Road 24, Madera - CA - USA
}

\begin{abstract}
An experiment was carried out from 2005 to 2008, to calibrated NIR-based instrumentation and explore within field grape quality variability and map potential grape quality descriptors along vineyards, as a subsidy for differential harvesting,. The quality indicators (anthocyanin content, $p H$, titratable acidity and soluble solids) were subject to geo-spatial modeling. Subsequently, the data set was utilized to delineate "within-field" grape quality zone and to determine the timing of the harvest. Differential machine harvesting was implemented and segregation of wine grapes was done "on-the-go". The approach for field prediction of grape quality parameters and zone delineation allowed for separated fermentation for at least two wine styles.
\end{abstract}

Key words: NIR spectroscopy, grape, wine

\section{INTRODUCTION}

Grape yield and quality can vary from berry to berry, cluster to cluster and vine to vine basis, so "within-field" variability can be expected as a result of interaction between the plant, soil and climate, as reported by Bramley and Hamilton (2005), Bramley (2005) and Santos et al. (2008). Therefore, an opportunity can exist for grape quality management based upon reasonable variability of grape quality parameters, which would allow for meaningful contrasting zone delineation and selective harvesting for a separated fermentation, aiming at wine improvement through better source allocation. An intensive sampling, which is required for the discussed task would be necessary as to better account for field variability. Assessing grape quality parameters in the field based upon laboratory analysis is nowadays restrictive, as this involves laborious and time consuming procedures. Therefore, fast, reliable and portable analytical techniques are highly desirable for grape quality assessment, either for the winery or field determinations. As these techniques improve, so does the ability of exploration of the "within-field" variability for winegrapes.

During recent years, developments in spectroscopic instrumentation and chemometrics software (Workman and Burns 2001) have made it possible to rapidly perform multivariate analysis, correlating spectral data with quantitative chemical data. These developments have led the way for a much broader use of spectroscopy as an accurate

*Author for correspondence: odairsan@iac.sp.gov.br 
predictor of chemical concentrations of substances within a wide range of media (Wold et al. 2001; Bjorsvik and Martens 2001).

Analysis using NIR spectroscopy has several desirable characteristics. These include: nondestructive sampling, rapid data acquisition (7-10 seconds per scan) and sensitivity to a wide range of molecules containing $\mathrm{C}-\mathrm{H}, \mathrm{N}-\mathrm{H}, \mathrm{S}-\mathrm{H}$ and $\mathrm{O}-\mathrm{H}$ bonds (Ciurczak 2001). Mathematical modeling using data obtained with reference methods and spectral information can be performed using multivariate analysis (Kaye and Wample 2005; Gishen et al. 2005).

Amongst the parameters for grape quality characterization, the content of polyphenols and anthocyanin has been cited to have relevant correlation to the quality and the intensity of the aroma of wine (Francis et al. 1999). Soluble solids, $\mathrm{pH}$ and titratable acidity (TA) have also been, traditionally, used as indicator of grape quality in the wine industry.

The goal of this investigation was to calibrate and apply near infrared spectroscopy to assess the spatial behavior of grape quality parameters along the vineyards and promote differential mechanical harvesting, according to quality zone delineation.

\section{MATERIAL AND METHODS}

\section{Vineyards}

Data for NIR Calibration and for field prediction (grape quality parameters) were collected along vineyards of Cabernet Sauvignon, Syrah and Merlot in the Sao Joaquin Valley (SJV-USA), during the 2005 and 2007 growing seasons. The data was acquired from berry set until harvest. Vineyards were irrigated and the soil and plant management was applied according to traditional recommendation for the region.

The SJV is characterized by hot and dry summers with a persistently high vapor pressure deficit throughout the growing season. It is a dry climate of middle latitudes, with most of the rain occurring in the winter. According to Critchfield (1983), it is framed as $B W k$ (Dry, desert vegetation is dominant, desert climate of middle latitudes), following the Koeppen climatic classification. In the California classification system determined by Winkler, it is in Region V, signifying the highest possible number of Growing Degree Days (GDD) per growing season (Winkler et al. 1974).

\section{NIR instrumentation and calibration}

The levels of anthocyanin $(\mathrm{mg} / \mathrm{g})$, soluble solids ( ${ }^{\circ}$ brix), $\mathrm{pH}$ and titratable acidity $(\mathrm{g} / \mathrm{L})$ were analyzed in the field, by using a (portable) handheld NIR instrument (NIRS), namely Luminar 5030 system (Brimrose Corp, MD, USA). The system's NIR monochromatic beams were generated based upon Acoustic-Tunable-OpticFilter (ATOF) technology (Workman and Burns, 2001). AOTF uses sound waves to vibrate an acoustically sensitive crystal inside the spectrometer. The vibrating crystal precisely fractionates a light source into specific wavelengths. Those wavelengths can then be focused upon a surface. NIR spectral reflectance can then be measured and expressed as transmittance. The spectral range used was 1100 to $2300 \mathrm{~nm}$ in transmission mode, with a wavelength increment of $2 \mathrm{~nm}$. The signal to noise ratio was increased by scanning this spectral range 100 times per measurement and averaging all the acquired spectra. After acquisition, the spectral data was converted to absorbance.

During the 2003 to 2005 growing seasons, over 2400 samples of wine grape berries were scanned with the NIRS and subsequently processed to determine the soluble solids, $\mathrm{pH}$ and $\mathrm{TA}$ values using a refractometer, $\mathrm{pH}$-meter and titration procedures, respectively. Soluble solids were measured for individual berries, while $\mathrm{pH}$ and TA were determined from combined samples of 50 to 100 berries.

Anthocyanins content was quantified using Iland extraction technique (Iland et al. 2004). Ninety six samples were used for build the model for anthocyanin.

\section{Chemometrics and data analysis}

Acquisition and transformation of all spectral data (lab calibration and in-field prediction thereafter) were processed using Brimrose Snap 2.04 ${ }^{\circledR}$ software (Brimrose, Md, USA). Chemometric analysis and statistical interpolation of the transformed spectra were accomplished using CAMO Unscramble $8.0^{\circledR}$ software (CAMO, Norway).

Calibrations were developed using partial least square regression (PLS) (Bjorsvik and Martens 2001).

Cross validation was used to estimate the prediction error by splitting all the samples into groups. One group was reserved for validation and the remaining groups were used for calibration. 
The process was repeated until all the groups were used for validation once. No pre-treatments (first or second derivative, etc) were applied to the spectral data before calibration development.

The performance of the model was verified by the standard error of cross-validation (SECV) and correlation coefficient (CC). The best calibration model was selected based on the minimum SECV. For data predicted in the field, spatial variability of grape quality parameters was investigated through the semi-variogram analysis (Isaaks and Srivastava 1989). The magnitude of variability in the in-field predicted data was analyzed based upon univariate statistics (coefficient of variation), variogram parameters (Nugget, Sill and range) and the Cambardella Index (CI) (Cambardella et al. 1994). Cambardella Index allows to verify the level of spatial dependency eventually present in the data (strong to weak).

\section{Field sampling scheme}

In the growing season of 2005, two vineyards of Merlot were selected to test grape parameter prediction and mapping. Study was also conducted on grape quality variability in the field, based upon the calibration performed in the laboratory. For the first site, namely "Joe Cotta", 48 samples were taken in the north side of the field and 46 samples were taken in the south side. Therefore, an area of 8.38 ha was divided evenly in two blocks and sampled at 94 points, using a $49 \mathrm{~m} \times 31.5 \mathrm{~m}$ grid. The division in two blocks was necessary to avoid a non-Merlot spot in the field (rootstocks area). A second site, namely "Schaeffer" of 12.51 ha was sampled at 84 points in a $45 \mathrm{~m} \times 33 \mathrm{~m}$ grid. Prior to grape sampling, in both described sites, the spectra of three clusters (three berries per cluster) in each vine were acquired per sampling point.

The entire sampling scheme was based on a metric scale set up based upon the vine spacing.

For the collection of spectra in the field, the NIR hand held unit was equipped with a battery pack and transported on the front end of an all-terrain vehicle (ATV).

The values of anthocyanin ( $\mathrm{mg} / \mathrm{g})$, titratable acidity $(\mathrm{g} / \mathrm{L}), \mathrm{pH}$ and soluble solids values were estimated in the field and their structure of variability were verified.

Based on the lessons learned in the 2005 season, in 2007, an intensive sampling was implemented; fruit quality mapping and differential harvest were executed in the block of Cabernet Sauvignon, located in Madera, CA (USA). Anthocyanin (mg/g of fresh weight) was the parameter used to map the quality zones; cluster counting, cluster weight and yield/area were also gathered.

Te sampling was performed in two different dates, just prior to the harvest. The first sampling was done in the whole area ( 80 ha) on $24^{\text {th }}$ August and 1300 vines were sampled. This was repeated on $8^{\text {th }}$ September, but only in half area (40 ha), comprising 698 sampled vines. The vineyard had a vine spacing of $3 \mathrm{~m} \times 2.26 \mathrm{~m}$. The calculated number of vines in the whole experimental area was 45,318 . The Grid sampling was around $15 \mathrm{~m} \mathrm{x}$ $20 \mathrm{~m}$ in both sampling trails.

A total of ten clusters from every data vine were collected. Of the ten clusters, five clusters were selected from the upper $50 \%$ of the canopy and five clusters from the bottom portion. There was an effort to proportionally sample the clusters from each portion of the canopy based on cluster size. Prior to cluster sampling, several vines were completely harvested and the percentage of large, medium and small clusters was determined. The combined information was used to establish the sampling protocol. This procedure was strictly employed to acquire the samples from each vine. Fruit samples were placed in the plastic bags, weighed, and placed in ice chests to be transported to the lab for anthocyanin analysis. Approximately $400 \mathrm{~g}$ of fruit was collected from the 10 clusters for anthocyanin analysis by using a Zeiss Corona 45 VISNIR 1.7 spectrometer (Carl Zeiss MicroImaging, Inc, Thornwood, NY). The resulting anthocyanin values in conjunction with their corresponding GPS coordinates and GIS software were used to establish different quality zones within the vineyard. The assessed cluster counting and cluster weight was used to conclude about yield per vine in the vineyard.

An multispectral image was collected by a an aircraft in the area, in order to map the wine grape biomass variation. For this, a flight was run in the field when the crop was around bloom.

A wine grape harvester Korvan 3016XL (Oxbo Corporation, Byron, NY) was used in the experiment. The harvester had two OTR conveyors. The differential harvesting was accomplished by controlling the direction of motion of the conveyor which delivered the harvested fruits either to the left side or right side of the harvester. The spatial Management System (SMS) Basic software (Agleader Technologies, Ames, IA) was used for fruit quality coding. INSIGHT $^{\mathrm{TM}}$ monitor, Direct Command ${ }^{\mathrm{TM}}$ liquid 
application control hardware (Agleader Technologies, Ames, IA) was installed in the harvester. A Raven 100S GPS receiver was attached. The total circuit consisted of a monitor that controlled the master conveyor belts of the wine grape harvester through a adapted "liquid product control module hardware" that sent a control signal to activate or deactivate a "relay switch" for directing the master conveyor, thereby controlling the flow of wine grapes into the assigned gondolas (left or right). Further details on the modification of the harvesting procedure in order to arrange for differential harvest in the area was as described by Sethuramasamyraja et al. (2007).

As the half area was representative for the grape quality variation, differential harvesting was implemented only in the half south part of the field (40 ha) two weeks after the last sampling.

\section{Post-harvesting and wet chemistry}

Field harvesting was performed separately by zones, according to the established levels of anthocyanin provided through geo-statistical and GIS analysis.

The harvesting was a "two steps" procedure for picking up the grapes. At first, there was a start in one side of the area and the harvesting headed to the center of the area. At this step, all the grapes were taken to the winery, namely Paul Masson (PM). Later,the mapping was re-scaled so that the selection of the grapes was more rigorous, as the transition for considered high quality grapes was raised slightly. At this step, the harvesting started in the opposite side of the area and headed to the center. All the grapes collected in this step were sent to a winery, namely Woodbridge (WB).

A total of 284.13 tons of grapes was sent to PM and 193.60 tons were sent to WB. The lots were fermented in separate tanks. They were named as high quality (HQ) grapes harvested in the zones of higher anthocyanin and NQ - Normal quality for the rest of the grapes.
After field harvesting and grape crushing, subsamples of all the juice under fermentation were sampled and prepared by centrifugation and filtration for color analysis by using spectrophotometer in October 2007. In March 2008, the tanks were sampled again and phenolic pigmentation was analyzed according to Adams and Harbertson (1999).

\section{RESULTS AND DISCUSSION}

Figure 1 shows the results from the calibration performed in the lab for the near infrared instrumentation. Several samples were used for soluble solids, $\mathrm{pH}$ and TA modeling (Table 1). For anthocyanin, 96 samples were used. The correlation coefficients for the prediction models (Table 1) revealed the level of exactitude and the standard error of cross validation (SECV) indicated the level o precision of the models.

In spite the slightly raised values of SECV, the correlation coefficient revealed strong relationship between the measured and estimated variables. Results from the similar instrumental calibration for soluble solids values were reported by Desseigne et al. (2003). The author also reported correlation coefficients varying from 0.90 to 0.93 and mean square error of 0.95 to 1.26 for that variable. Until now, no similar report has been made on the others models (TA, $\mathrm{pH}$, anthocyanin), by using similar procedure, but the values for correlation coefficient for these variables seemed reasonable for extrapolation. Particularly, anthocyanin could have the calibration improved by adding new samples in the set of calibration in the future.

Anthocyanin seemed to be most promising among the all the variables analyzed, for being determined by calibrated NIRS as the color analysis was a good indicator of quality (Francis et al. 1999) and due to the difficulty in making this variable available on time for decision making in the wine grape industry.

Table 1 - Descriptive statistics for the calibration performed for the NIRS system.

\begin{tabular}{ccccc}
\hline & N of samples & CC & SECV & BIAS \\
\hline Soluble solids $\left({ }^{\circ}\right.$ Brix) & 2.454 & 0.92 & 1.9 & $-3.10^{-6}$ \\
$\mathrm{pH}$ & 2.415 & 0.86 & 0.16 & $8.31 .10^{-8}$ \\
$\mathrm{TA}(\mathrm{g} / \mathrm{L})$ & 2.598 & 0.86 & 1.89 & $-1.09 .10^{-6}$ \\
Anthocyanin $(\mathrm{mg} / \mathrm{g})$ & 96 & 0.82 & 0.11 & $2.73 .10^{-8}$ \\
\hline
\end{tabular}

Note: TA (titratable acididy); CC (correlation coefficient); SECV (standard error of cross validation) 


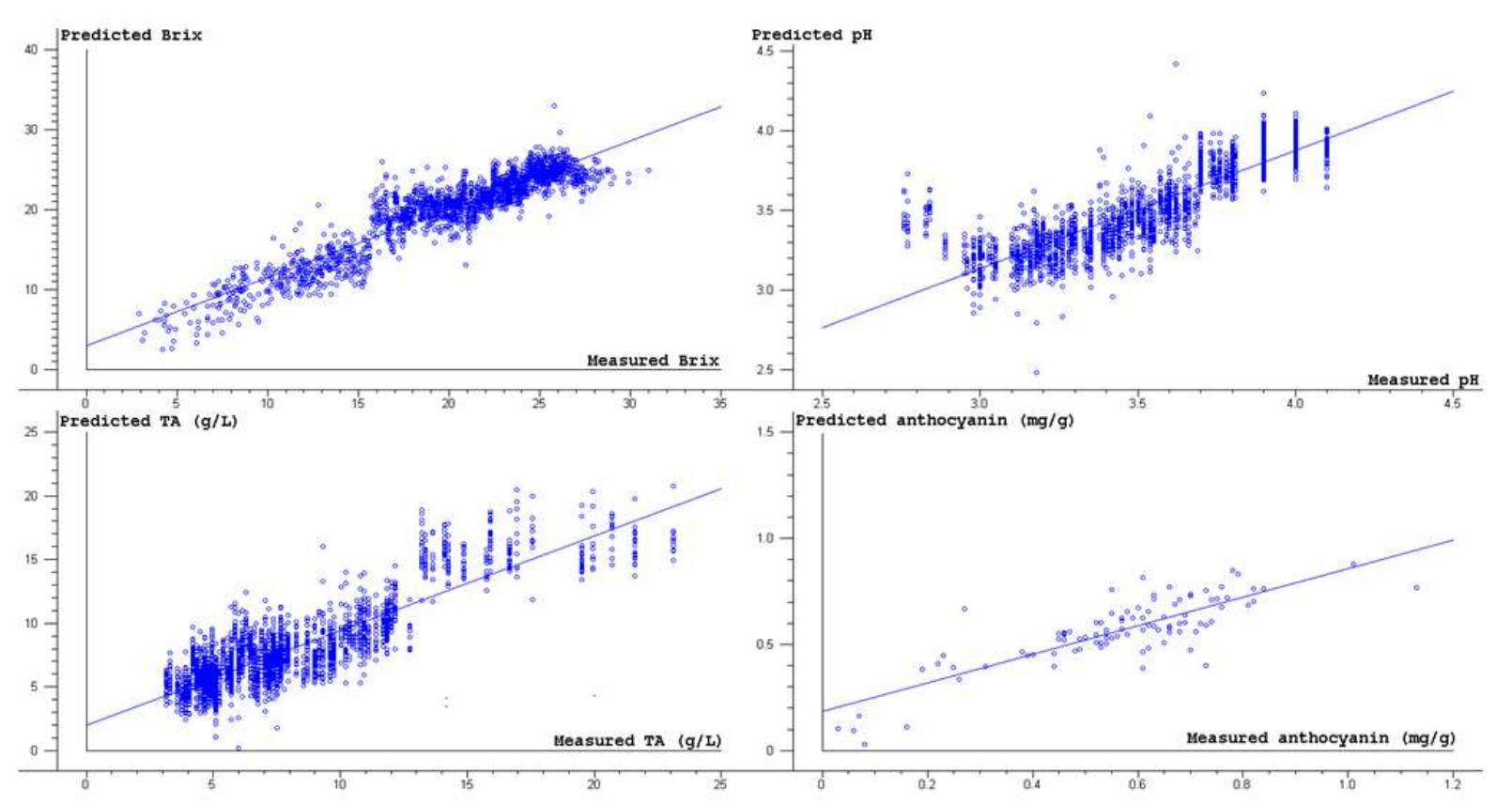

Figure 1 - Results from the comparison between estimated grape quality parameters by NIRS and measured values obtained by reference methods, in the calibration procedure.

Results from the spatial analysis made on the sampling scheme are presented in Figure 2. The spatial analysis showed the differences in different parts of vineyard. The CV value showed the variation in the whole set of data, independent of the field coordinates. Both analyzed in tandem could give clear understanding to the magnitude of variability in the field.

After the analysis of the variograms, it was found thatfor all the fields sampled for grape quality, the variograms were systematic flat. The $\mathrm{CI}$ index revealed that all quality parameters for the grapes were weak to moderate spatial dependence. Anthocyanin had moderate spatial dependence in the Cotta south and in the Shaeffer site, which made it more predictable than $\mathrm{pH}$ and TA. However, in general most of the models had high values for the sill (Table 2), which meant that a considerable level of randomness was present in the data for the grid used in the sampling scheme. Most of models had moderate spatial dependency as CI index was between 25-75.

Soluble solids showed the most unfavorable structure when considering the opportunities of management into precision viticulture concept. It had the least coefficient of variation and the variogram showed weak spatial dependency in all the areas analyzed. Therefore, soluble solids had a higher level of uncertainty for being predicted in the field, based on the present sampling scheme.

The $\mathrm{pH}$ and $\mathrm{TA}$ values were interesting in both the blocks in the Joe Cotta site. They showed pure nugget effect (total randomness) and light spatial structure occurring in the opposite blocks. For all the cases of pure nugget effect, the mapping was uncertain because abrupt variation occurred with the increasing distance in the area and the variable could not be reasonably predicted in un-sampled locations. This indicated a total lack of autocorrelation for that variable.

Bramley (2005) working with different varieties has reported similar behavior for the variogram analysis for an even smaller sampling grid. For the same set of variables, Bramley (2005) reported a moderate level of spatial dependency and considerable level of randomness in the data. Continued research with the NIRS can allow for better understanding of the spatial behavior of such variables in different fields and growing seasons. 
Joe Cotta site, block north
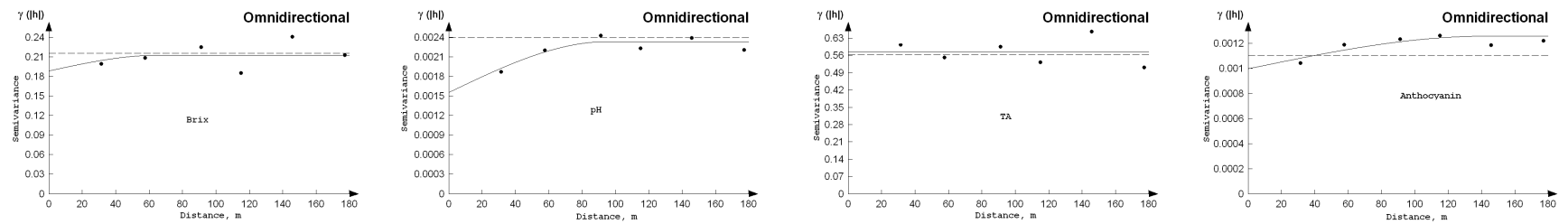

Joe Cotta site, block south
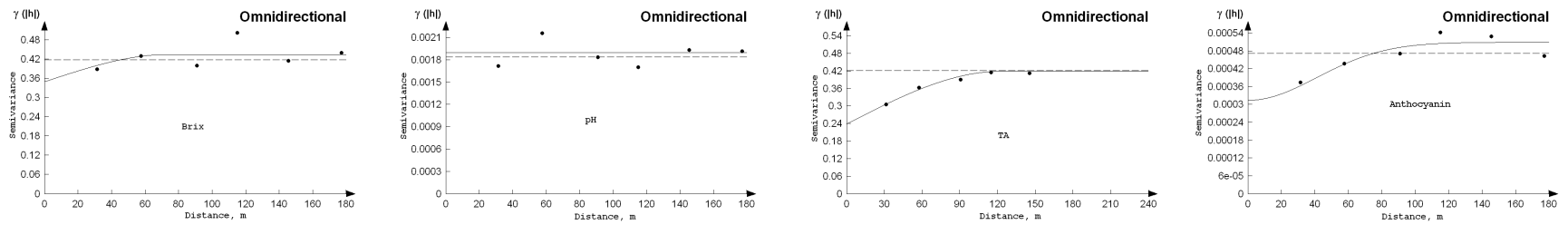

Shaeffer site
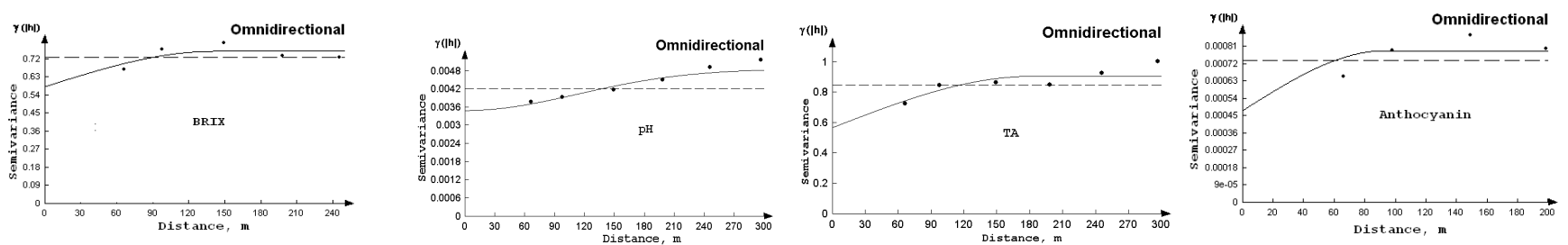

Figure 2 - Variogram analysis for three different areas, sampled for grape quality parameters (soluble solids (Brix), pH, titratable acidity (TA) and anthocyanin) by near infrared spectroscopy.

Table 2 - Descriptive spatial statistics for three sites sampled for grape quality parameters.

\begin{tabular}{lccccc}
\hline & Nugget & Sill & Range, $m$ & CV \% & CI \\
\hline Joe Cotta North & & & & & \\
Soluble solids ('Brix) & 0.189 & 0.024 & 64.28 & 5.42 & $88.73 \mathrm{~W}$ \\
pH & 0.00156 & 0.00078 & 95.4 & 3.19 & $66.67 \mathrm{M}$ \\
TA (g/L) & - & - & - & 21.47 & \\
Anthocyanin (mg/g) & 0.000999 & 0.00026 & 142 & 13.08 & $79.35 \mathrm{~W}$ \\
Joe Cotta South & & & & & \\
Soluble solids ('Brix) & 0.349983 & 0.085 & 73.8 & 5.41 & $80.46 \mathrm{~W}$ \\
pH & - & - & - & 3.27 & \\
TA (g/L) & 0.239967 & 0.17998 & 122.4 & 19.31 & $57.14 \mathrm{M}$ \\
Antocyanin (mg/g) & 0.000314 & 0.00019 & 100.79 & 11.88 & $62.30 \mathrm{M}$ \\
Shaeffer & & & & & \\
Soluble solids ('Brix) & 0.584 & 0.168 & 120 & 6.56 & $77.66 \mathrm{~W}$ \\
pH & 0.0035 & 0.00137 & 273.63 & 4.18 & $71.87 \mathrm{M}$ \\
TA (g/L) & 0.5694 & 0.3381 & 181.04 & 17.94 & $62.74 \mathrm{M}$ \\
Anthocyanin (mg/g) & 0.00048 & 0.00031 & 92.83 & 11.30 & $60.75 \mathrm{M}$ \\
\hline
\end{tabular}

Note: TA (titratable acidity); CV (coefficient of variation); CI (Cambardella index); W, M: (weak and moderate levels of spatial dependency).

Taylor et al. (2005) reported coefficients of variation for grape yield in the range of 21.83 to $50.66 \%$, when comparing a set of vineyard in Europe to the ones in the New World. A comparison of these values to the coefficients of variation depicted in the Table 2 revealed that grape yield had more pronounced variation in the field. An interesting point in this matter would be to verify the existing relationship between the identified zones in the field, based upon a set of soil-water variables plus yield, and the information plan built for grape quality. This could allow 
benefiting from any existing positive relationship among the grape quality and delineated zones. Moreover it's a meaningful way of interference in the system, so as to attempt to increase the grape quality by managing water, pruning and nutrition in a zonal concept, provided that the reasonable spatial contrasts for the most influential variables on the yield and quality could exist and result in persistent pattern of grape quality along the years. Figure 3 shows the results for the frequency of distribution for the sampled anthocyanin in the different sampling scheme applied to the area of

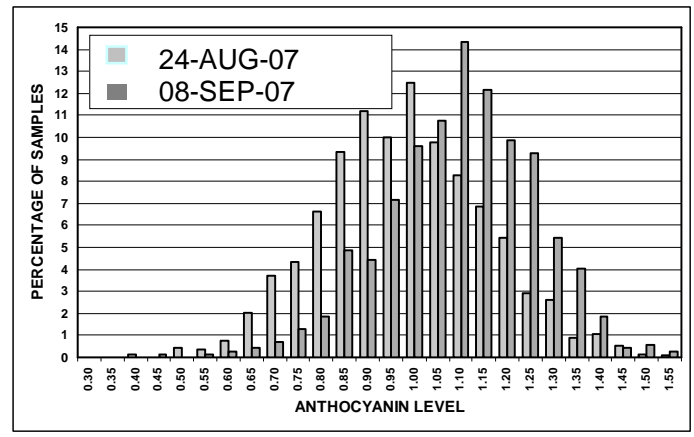

Cabernet Sauvignon variety in 2007 season. The data showed a histogram shaped to a normal distribution. The data had reallocated itself due the maturation process that took place in the field. Therefore, the frequency values had changed proportionally, as the grape had proceeded with the ripening. The data suggested that both the sampling data could be used for the mapping, as the zone delineation would not be affected, if the relative location for high (HQ) and low values (NQ) were kept the same.

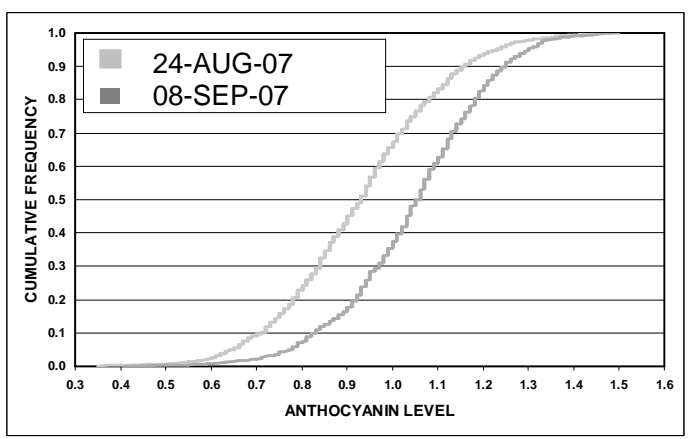

Figure 3 - Histogram and Cumulative Frequency for two sampling dates, in an area of grape Cabernet Sauvignon.

Figure 4 shows the results from the sampling scheme applied in the Cabernet Sauvignon plot in 2007 growing season. Levels of anthocyanin and yield were put together, so one could compare the relationship between each other. This showed correlation in a considerably area based upon a very intensive sampling scheme and was a good opportunity to see how the variables behaved in an open field.

The image in the center represented the variation in vigor, estimated by color composition. Evidently a large part of the area had increase in anthocyanin content along the field, followed by decrease in yield levels. A comparison between the yield, anthocyanin and vigor levels showed that they were visually correlated

This showed that there was negative correlation between the anthocyanin and vigor. This has been supported by other studies (Cortell et al. 2005). Therefore, until high amount of anthocyanin was present, lower, levels of vigor was expected.

Visual comparison showed a positive correlation between the yield and vigor while anthocyanin levels had a negative correlation with vigor and yield. In fact, anthocyanin levels seemed to be associated to the areas of decreasing vigor. This was very clear for the south part of the image which had higher levels of anthocyanin corresponding to lower levels of vigor. This was also true for the north part of the image.

In spite of this, the vigor image, as shown in the Figure 4, could be used in order to fine tune any differential harvesting procedure. Figure 5 showed that regardless of higher levels of simultaneous sampling of anthocynin with low yield, there was a considerable amount of vines with high values of anthocyanin and high yield in the same vine. In fact $16.4 \%$ of points in the area $(16.4 \%$ of the vines) had higher levels of anthocyanin and also higher levels of grape yield. This showed that a complex interaction occurs in the field and makes the relationship between the levels of anthocyanin and yield not always to be a linear correlation.

A calculation of NDVI (Lamb et al. 2004) from the extracted pixel values was implemented on vigor image (Fig. 4) and further analysis allowed, in a pixel by pixel comparison among vigor, anthocyanin and yield (Fig. 6). The correlation coefficient for the relationship between the vigor an anthocyanin were shown to be week, which was in accordance to the image, where only sometimes coincidences were noticed. 

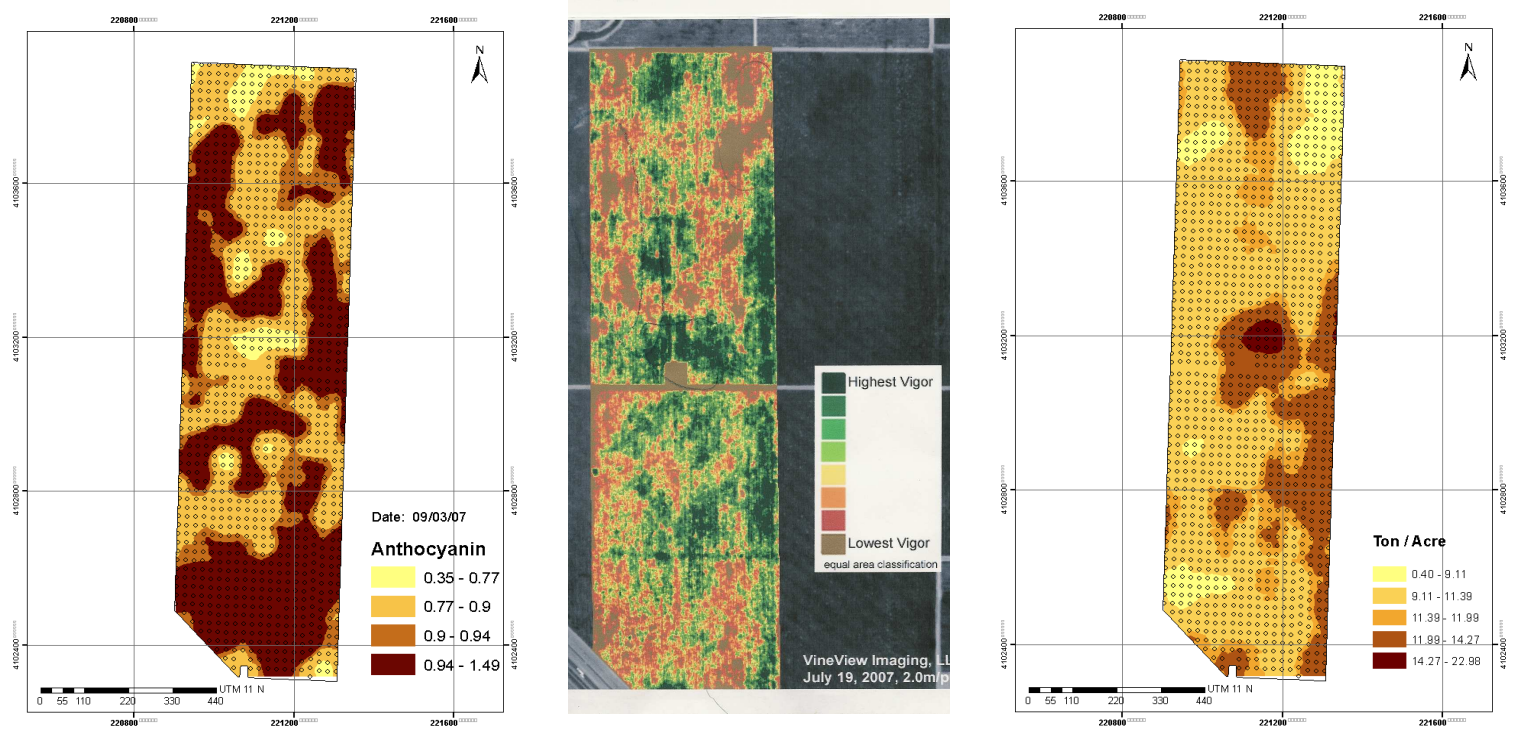

Figure 4 - Sampling points and kriged anthocyanin and yield surfaces and vigor image in a 80 ha area of Cabernet Sauvignon.
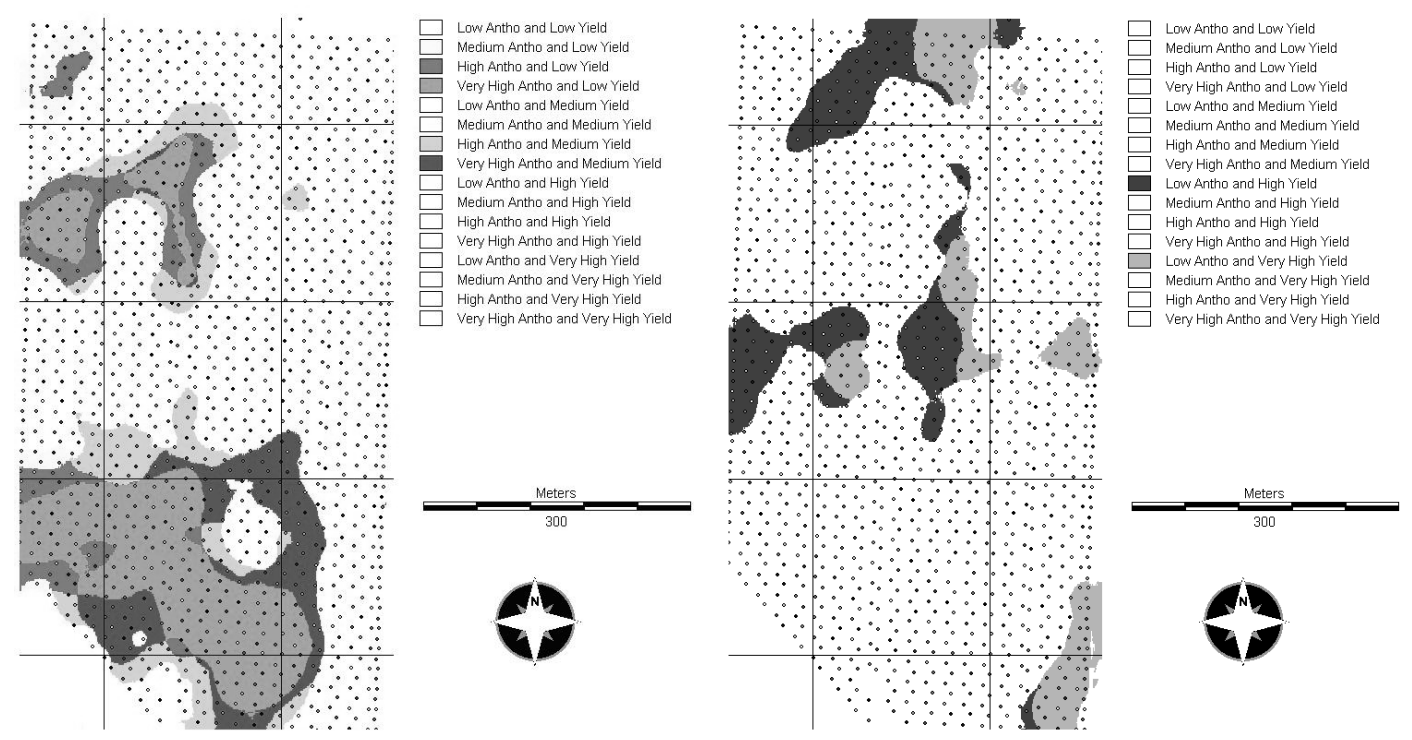

Figure 5 - GIS-Crosstabulation of the spatial distribution of anthocyanin (Antho) (mg/g) and yield (kg/vine) in a Cabernet Sauvignon area, in the Central Valley (CA/USA), in the growing season of 2007. 

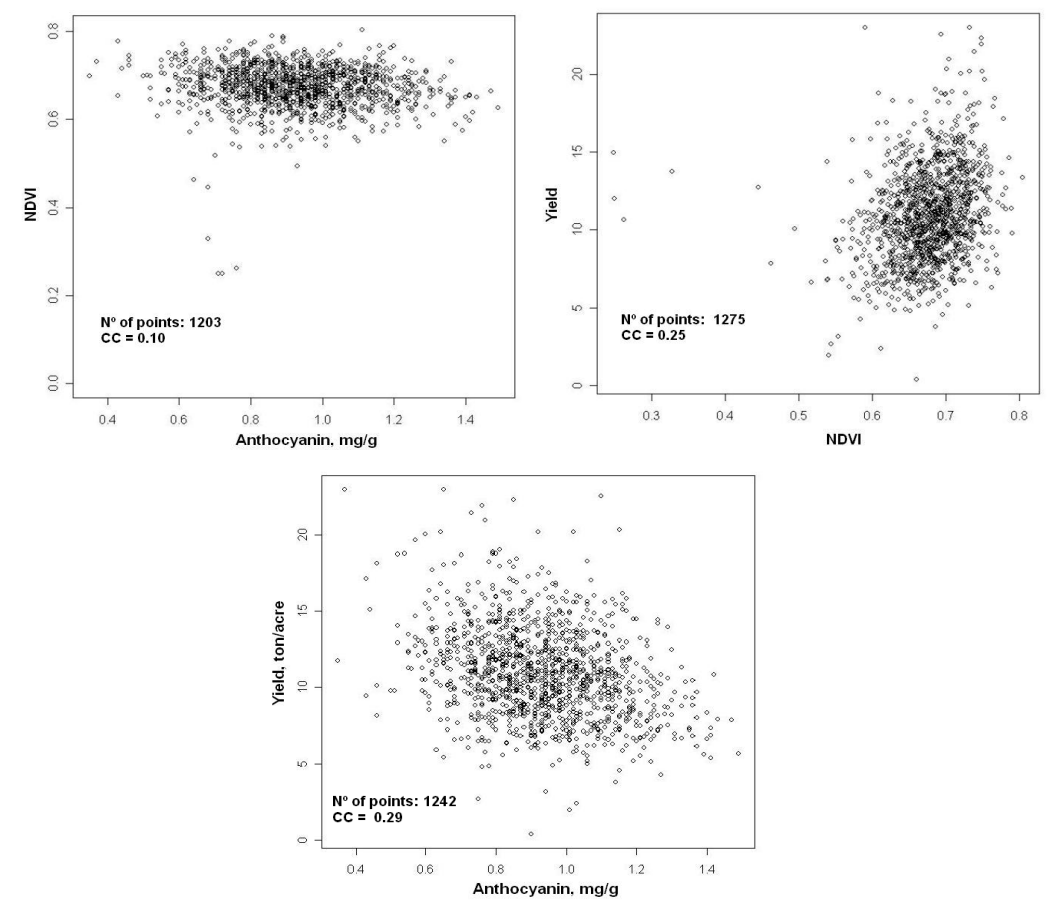

Figure 6 - Scatter diagram for the relashionship among extract NDVI from the Vigor Image and corresponding pixel values for yield and anthocyanin.

In fact, as far as the use of color composition image, or NDVI-based image would ease the process of quality zone delineation, some other authors have also showed weak correlation between the NDVI-based image and grape quality parameters. Mendez-Costabel (2009), working with Merlot indicated that the fruit and wine quality components analyzed did not show a clear relation with the NDVI, so, the use of average NDVI values per row within the vineyard block was not accurate for separating the fruit quality differences. In fact, vineyards presents a challenge in the application to the remote sensing technology, mainly due to a) discontinuous nature of grapevine canopy; b) moderate cover with a prominent background and shadow influence on the measured reflectance signal.
Nevertheless, the fine tuning of this technique could lead to improve its use in the precision viticulture. This would be very important as the sampling, in the sense of this work was still very time and money consuming.

The Figure 7 shows the final handling of the mapping in order to implement the differential harvest. Figures $7 \mathrm{a}$ and $7 \mathrm{~b}$ depict a different scaling in the levels of anthocyanin. The data were rearranged in the Figure $7 \mathrm{~b}$ in such way that a more rigorous selection was made and the "threshold" for the transition for high quality was raised slightly. As a result, Figure $7 \mathrm{~b}$ represented a search for the "considered" best in quality along the field, or the "cream crop". As the area harvested in the left part of the Figure $7 \mathrm{a}$ and the right part of the Figure $7 \mathrm{~b}$, the lots of grapes was sent to two different wineries. 

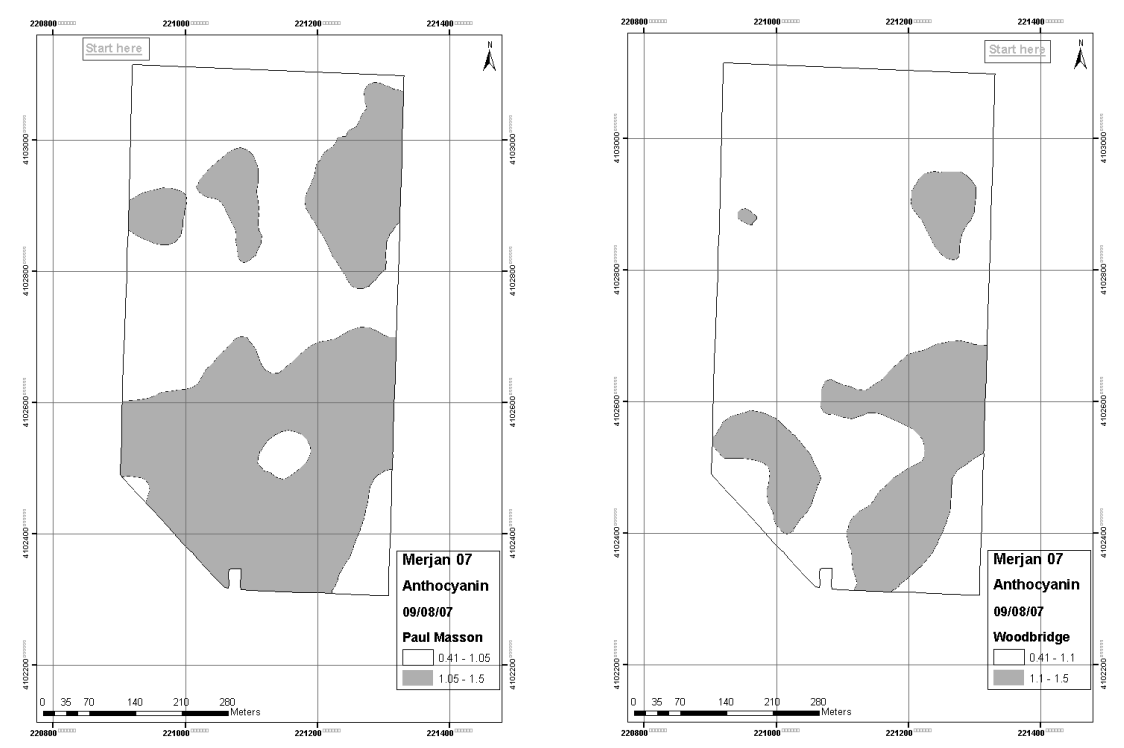

Figure 7 - Final map for machine guidance in a differential harvesting trial, in a plot of 40 ha Cabernet Sauvignon, in Madera, CA, during the growing season 2007/2008.

Table 3 shows the results for the colormetrics performed in the grape must at the winery, just after the harvest for the sampling in the tanks under fermentation. For the lot of grapes sent to PM Winery, the color intensity revealed that the zones considered as high quality were in accordance to the planned zones, as the value for HQ was higher than NQ. This was also true on considering the "free run" and the "first press" fraction. The lots of grapes sent to the WB winery showed that the relation between HQ and NQ was in accordance to the delineated zones in the field. This first analysis (Table 3) revealed that the sampling and kriging of the field in order to achieve the quality zone delineation was successful up to this point.

Figure 8 shows the analysis implemented in the wines on $24^{\text {th }}$ March, 2008. The sampling in the tanks for both the wineries revealed that for the PM Winery, the tanks fermenting the "free run" the total phenolics and tannins were higher for HQ zones, in spite that the anthocyanin values were pretty similar between the both zones. For the tanks containing the first press and free run, the levels of anthocyanin were higher for NQ tanks. However, the tannins and total phenolics were in reverse order.

This showed that increased time of fermentation resulted higher extraction of anthocyanin for the HQ. it was also possible that the bounding and polymerization of tannins caused it to decrease in a more pronounced manner in the HQ tanks. Due to this, the, non- phenolics were higher for HQ. However, this was not influenced by the use of anthocyanin as a quality indicator.

$\underline{\text { Table } 3 \text { - Colormetrics from the fementing tanks sourced in a differential harvesting trial. }}$

\begin{tabular}{lcccc}
\hline Treatment & $\begin{array}{c}\text { Absorbance } \\
\text { A520 }\end{array}$ & $\begin{array}{c}\text { Absorbance } \\
\mathbf{A 4 2 0}\end{array}$ & $\begin{array}{c}\text { Intensity } \\
\mathbf{A 5 2 0 + A 4 2 0}\end{array}$ & $\begin{array}{c}\text { Hue } \\
\mathbf{A 5 2 0} / \mathbf{A 4 2 0}\end{array}$ \\
\hline Paul Masson (HQ) "Free run" & 0.87 & 0.522 & 1.392 & 1.666 \\
Paul Masson (NQ) "Free run" & 0.821 & 0.531 & 1.352 & 1.546 \\
Paul Masson (HQ) "Free run" + $1^{\text {st }}$ press & 1.074 & 0.639 & 1.713 & 1.681 \\
Paul Masson (NQ) "Free run" + 1 $^{\text {st }}$ press & 0.959 & 0.605 & 1.564 & 1.585 \\
Woodbridge (HQ) "Free run"” only & 1.377 & 0.874 & 2.251 & 1.575 \\
Woodbridge (NQ) "Free run" only & 1.285 & 0.796 & 2.081 & 1.614 \\
\hline
\end{tabular}




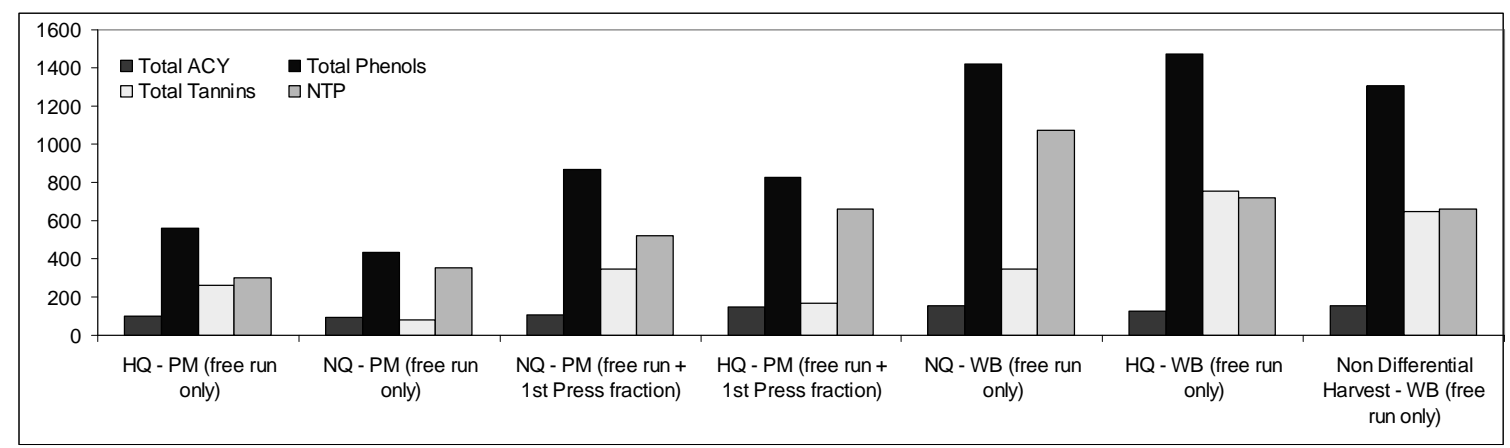

Note: ACY (anthocyanin)

Figure 8 - Analysis for quality indicators in the finished wines, in 2008, originated from a differential harvesting procedure, in a Cabernet Sauvignon area.

For the WB winery, the results from the fermenting tanks after five months revealed values higher than PM winery tanks (Fig. 9). This was also true when compared even to the nondifferential harvesting tanks. Total phenolics and total tannins were higher for the HQ zones, showing that the planned zones were in accordance with the results of the fermenting tanks. As the woodbridge was contemplated with the considered "cream crop", due to the more rigorous division in the threshold for anthocyanin, its could be concluded that the mapping area and the search for the best grape quality was achieved in this work. In general, wet chemistry analysis performed in the grape must and in the fermenting tanks showed the agreement between the expected values for the delineated quality zones and the final results obtained afterwards.

It might be possible that very intensive sampling used in this work would not be feasible in many cases, as the costs would be restrictive for grape quality segregation in the field. However, as the sampling techniques and technology develop together, it could be expected to achieve faster samples at lower cost.

\section{CONCLUSIONS}

In all vineyards NIR-based data were acquired successfully. The approach to calibration and field prediction of grape quality parameters resulted in the harvesting and separated fermentation of at least two wine styles.
The separation of the best quality crop in the field was done by intensive sampling and differential harvesting, and proved accurate by wet chemistry. The trend of high yield and high quality did not have always a linear negative correlation in the field due to the complex interaction among the variables influencing the vine growth and development.

\section{REFERENCES}

Adams DO, Harbertson JF. Use of alkaline phosphatases for the analysis of tannins in grapes and red wines. Am J Enol Vitic. 1999; 50: 247-252.

Bjorsvik HR, Martens H. Calibration of NIR instruments by PLS regression. In: Burns D, Ciurczak EW, editors. Handbook of Near-Infrared analysis. New York: Marcel Dekker; 2001. p. 185-208.

Bramley RGV. (2005). Understanding variability in winegrape production systems; 2 . within vineyard variation in quality over several vintages. Aust $J$ Grape Wine Res. 2005; 11: 33-42.

Bramley RGV, Hamilton RP. Understanding variability in winegrape production systems; 1 . within vineyard variation in yield over several vintages. Aust J Grape Wine Res. 2004; 10: 32-45.

Cambardella CA, Moorman TB, Novak JM, Parkin TB, Karlen DL, Turco RF, Konopla AE. (2004). Field-scale variability of soil properties in Central soils. Soil Science Soc Am J. 2004; 58: 1501-1511.

Ciurczark EW. Principles of Near-Infrared spectroscopy. In: Burns D, Ciurczak EW, editors. Handbook of Near-Infrared analysis. New York: Marcel Dekker; 2001. p. 4-7. 
Cortell JM, Halbleib M, Gallagher AV, Righetti TL, Kennedy JA. Influence of vine vigor on grape (Vitis vinifera L. cv. Pinot Noir) and wine proanthocyanidins. Journal of Agric. Food Chem. 2005; 53 (14): 5798-5808.

Desseigne JM, Crochon M, Roger JM, Ballester JF, Boulet JC, Mazzolier J, Toussaint C. Spectrometrie proche infra rouge et appreciation de la qualite de la vendange. In: Eurovit: Proceedings: Contributed papers-abstracts; 2003 Oct; Montepellier, France. Montepellier: Eurovit; 2003. p. 168-172

Francis IL, Iland PG, Cyncar WU, Kwiatowski M, Williams PJ, Armstrong H, Botting DG, Gawel R, Ryan C. Assessing wine quality with the G-G assay. In: Australian wine industry technical conference: Proceedings: Contributed papers-abstracts; 1999 Oct; Sydney, Australia. Sydney: AWA; 1999. p. 1-10.

Gishen M, Dambergs RG, Cozzolino D. Grape and wine analysis - enhancing the power of spectroscopy with chemometrics. A review of some applications in the Australian wine industry. Aust J Grape Wine Res. 2005 (11): 296-305.

Iland P, Bruer N, Edwards G, Weeks S, Wilkes E. Chemical analysis of grapes and wine: techniques and concepts.Adelaide: Tony Kitchener Printings, Adelaide; 2004.

Isaaks E H, Srivastava RM.An Introduction to applied geostatistics. 1st ed. Oxford: Oxford University Press; 1989.

Kaye O, Wample RL (2005). Using Near-infrared spectroscopy as an analytical tool in vineyards and wineries. In: ASEV annual conference: Proceedings: Contributed papers-abstracts; 2005 July; Seattle, EUA. Seattle: ASEV; p. 1- 9.

Mendez-Costabel MP, Sanchez LA, Dokoozlian N, Campbell P. NDVI imaging as a tool for characterizing fruit and wine variability on two mechanically harvested Merlot vineyards in California. In: XII Congresso Latinoamericano de Viticultura Y Enología: Proceedings: Contributed papers-abstracts; 2009, Nov; Montevideo, Uruguay. Montevideo: AEU; p. 10-2.
Santos AO, Wample RL, Sachidhanantham, S, Sethuramasamyraja B, Kaye O. First attempts at instrumenting for grape quality-based harvesting procedure. XXXVII International Conference of Agricultural Engineering: Proceddings: Contributed papers-abstracts; 2008 Oct; Foz do Iguaçú, Brasil. Foz do Iguaçú: SBEA; 2008. p. 20-22.

Santos AO, Wample RL, Kaye O, Sachidhanantham S. Advanced streaming grape quality for precision viticulture. XXXVII International Conference of Agricultural Engineering: Proceedings: Contributed papers-abstracts; 2008 Oct; Foz do Iguaçú, Brasil. Foz do Iguaçú: SBEA; 2008. p. 23-25.

Sethuramasamyraja B, Sachidhanantham S, Yen M, Wample RL. Interpolation of wine grape quality indicators (Anthocyanin and Brix) and development of differential harvest attachment. In: ASAE Annual meeting: Proceedings: Contributed papers-abstracts; 2007 July; St. Joseph, EUA. St. Joseph: ASAE; 2007.

Taylor, J.; Tisseyre, B.; Bramley, R.; Reid, A. (2005). A comparison of the spatial variability of vineyard yield in European and Australian production systems. In: V European Conference on Precision Agriculture: Proceedings: Contributed papers-abstracts; 2008 Aug; Wageningen, The Netherlands. Wageningen: WR; 2008.

Winkler AJ, Cook JA, Kliewere WM, Lider LA. General Viticulture. 4th ed. Berkeley: University of California Press; 1974.

Wold S, Trygg J, Berglund A, Antti H. Some recent developments in PLS modeling. Chem intelligent lab systems. 2001; 58: 131-150.

Workman-Jr J, Burns DA. Comercial NIR instrumentation. In: Burns D, Ciurczak EW, editors. Handbook of Near-Infrared analysis. New York: Marcel Dekker; 2001. p. 53-70.

Received: December 09, 2010; Revised: May 15, 2011; Accepted: December 12, 2011. 